\title{
MicroRNA-376b promotes breast cancer metastasis by targeting Hoxd10 directly
}

\author{
NING AN ${ }^{1}$, XINMEI LUO ${ }^{2}$, MING ZHANG $^{1}$ and RUILIAN YU ${ }^{1}$ \\ ${ }^{1}$ Department of Oncology, Sichuan Academy of Medical Sciences \& Sichuan Provincial People's Hospital, \\ Chengdu, Sichuan 610072; ${ }^{2}$ State Key Laboratory of Biotherapy and Cancer Center, West China Hospital, \\ Sichuan University, Chengdu, Sichuan 610041, P.R. China
}

Received August 4, 2015; Accepted October 5, 2016

DOI: $10.3892 /$ etm.2016.3942

\begin{abstract}
Breast cancer is the most common malignant disease in women, and metastasis formed at distant anatomic sites was the major cause of cancer-related mortality. Thus, a novel therapy target and progression biomarker for breast cancer metastasis was necessary. microRNA (miR)-376b has been demonstrated to regulate angiogenesis; however, its role in cancer metastasis remains elusive. In the present study, the expression of miR-376b in normal breast tissue, JC and $4 \mathrm{~T} 1$ cells was determined by qPCR. Furthermore, in vitro and in vivo experiments were performed to determine the effect of miR-376b on breast cancer metastasis. The direct target of miR-376b was determined by the luciferase assay and western blotting. The results indicated that silencing of miR-376b by the miR-376-mimic significantly inhibited 4T1 cell migration and invasion in vitro. Lung metastasis was also evidently decreased after silencing of miR-376b in 4T1 cells. Moreover, the luciferase assay and western blotting identified that Hoxd10 is the direct target of $\mathrm{miR}-376 \mathrm{~b}$ during the regulation of breast cancer metastasis. To the best of our knowledge, the present study was the first to demonstrate the promoting breast cancer metastasis role of miR-376b by directly targeting Hoxd10. Therefore, it would be a novel therapy target and prognostic biomarker for breast cancer.
\end{abstract}

\section{Introduction}

Breast cancer is the most common malignant disease in women (1). Meanwhile, $90 \%$ of cancer-related mortality is caused by metastasis formed at distant anatomic sites by disseminated primary tumor cells (2). Breast cancer patients frequently develop metastases at multiple sites. Primary

Correspondence to: Dr Ruilian Yu, Department of Oncology, Sichuan Academy of Medical Sciences \& Sichuan Provincial People's Hospital, 32 West 2nd Section, 1st Ring Road, Chengdu, Sichuan 610072, P.R. China

E-mail: yuruilian@gmail.com

Key words: breast cancer, microRNA-376b, Hoxd10, metastasis breast cancer cells metastasize through the blood vessels to various distant organs, preferentially, to the lung, liver and bones (3). Surgery, radiation therapy and chemotherapy were the effective strategies used for treating primary tumor; however, these treatments have limited utility in curbing the metastatic spread of cancer cells, resulting in metastasis (4). Thus, novel therapy targets are required for treating breast cancer metastasis.

microRNAs (miRs) are short non-coding RNAs, 18-22 nucleotides long, which were discovered in mammals as a large class of evolutionarily conserved small noncoding RNAs (5). miRNAs regulate gene expression at the post-transcriptional level by targeting the 3'-untranslated region (UTR) of mRNA transcripts (6). Furthermore, miRNAs have emerged as powerful regulators of a wide range of biological processes through influencing protein translation. It was quickly recognized that miRNAs can be efficiently inhibited by antisense technologies for prolonged periods, which has increased the interest of researchers in the inhibition of specific miRNAs as a feasible therapeutic option for several diseases $(7,8)$.

A previous study has demonstrated that miR-376b regulates angiogenesis in middle cerebral artery occlusion (9). Meanwhile, miR-376-5p mediates the M3 subtype of muscarinic acetylcholine receptor-inducing cardioprotection (10). However, the role of miR-376b in regulating breast cancer metastasis remains elusive. In the present study, miR-376b expression in normal breast tissue (NBT), JC and 4T1 breast cancer cells was determined by qPCR. Furthermore, in vitro and in vivo experiments were performed to determine the effect of miR-376b on breast cancer metastasis. The direct target of miR-376b was determined by the luciferase assay and western blotting. The results indicated that silencing of miR-376b significantly inhibited 4T1 cell migration and invasion in vitro. Lung metastasis was also evidently decreased following silencing of miR-376b in 4T1 cells. Moreover, luciferase assay and western blotting identified that Hoxd10 is the direct target of miR-376b during the regulation of breast cancer metastasis. To the best of our knowledge, the present study was the first to demonstrate a role of miR-376b in promoting breast cancer metastasis. Therefore, it may be a novel therapy target and prognostic biomarker for breast cancer. 


\section{Materials and methods}

Cell culture and transfection. The JC and 4T1 breast cancer cell lines were obtained from American Type Culture Collection (Manassas, VA, USA). The cells were cultured in Dulbecco's modified Eagle's medium (DMEM) containing 10\% fetal bovine serum (FBS; Gibco; Thermo Fisher Scientific, Inc., Grand Island, NY, USA). All the cells were maintained in a humidified atmosphere containing $5 \% \mathrm{CO}_{2}$ at $37^{\circ} \mathrm{C}$. Cell transfection was performed using FuGENE HD Transfection Reagent (Roche Diagnostics, Indianapolis, IN, USA) according to the manufacturer's instructions. Briefly, 4T1 cells were seeded in 6-well plates at a density of $2 \times 10^{5}$ cells/well and cultured for $24 \mathrm{~h}$ to reach $70-80 \%$ confluence. A total of $2 \mu \mathrm{g}$ plasmids (encoding miR-376b-mimic, miR-376b and miR-NC) were diluted in $100 \mu \mathrm{l}$ DMEM without serum and $5 \mu 1$ FuGENE HD Transfection Reagent was added to the tubes containing diluted DNA. These were then mixed and the transfection complex was incubated for $15 \mathrm{~min}$ at room temperature, and then added to the 6-well plates.

Vector construction. The miR-376b and miR-376b-mimic expression plasmids and the negative control (miR-NC) were purchased from the Origene Company (Rockville, MD, USA). Plasmids were extracted using EndoFree Plasmid Giga kits (\#12362; Qiagen GmbH, Hilden, Germany) from DH5a (Genewiz, Suzhou, China) Escherichia coli transformants and stored at $-20^{\circ} \mathrm{C}$ until further use. The concentration was determined by measuring the A260/A280 ratio using an ND 2000 spectrophotometer (Thermo Fisher Scientific, Inc., Waltham, MA, USA).

Target prediction. The miRWalk database (http://www. ma.uni-heidelberg.de/ apps/zmf/mirwalk/) and other programs (miRanda, Sanger miRDB, RNAhybrid and Targetscan) were used variously for target prediction The online tool miRWalk 2.0 (http://www.umm.uni-heidelberg. de/apps/zmf/mirwalk/predictedmirnagene.html) was used to predict potential target mRNAs of miR-376b.

Quantitative polymerase chain reaction ( $q P C R)$. The total RNA was extracted from each experimental group using TRIzol reagent (Invitrogen; Thermo Fisher Scientific, Inc., Carlsbad, CA, USA) according to the manufacturer's instructions. The RNA concentration was assessed spectrophotometrically at $260 \mathrm{~nm}$ (ND 2000; Thermo Fisher Scientific, Inc.). In addition, reverse transcription was performed on the isolated total RNA using a Reverse Transcription kit (\#RR047A; Takara Bio, Inc., Otsu, Japan), and PCR was performed using a SYBR Premix Ex Taq kit (\#RR820A; Takara Bio, Inc.), according to the manufacturer's instructions. gDNA eraser $(1.0 \mu 1), 5 \mathrm{X}$ gDNA eraser buffer $(2.0 \mu \mathrm{l})$ and mRNA template $(2.0 \mu \mathrm{g})$ were added into one well. Then RNAase-free $\mathrm{H}_{2} \mathrm{O}$ was added to the final volume $(10.0 \mu \mathrm{l})$. The well was incubated at room for $5 \mathrm{~min}$. Reverse transcription was performed at $65^{\circ} \mathrm{C}$ for $5 \mathrm{~min}, 30^{\circ} \mathrm{C}$ for $10 \mathrm{~min}, 42^{\circ} \mathrm{C}$ for $10-30 \mathrm{~min}$ and $2^{\circ} \mathrm{C}$ for $3 \mathrm{~min}$. The PCR conditions were as follows: Denaturation at $94^{\circ} \mathrm{C}$ for $2 \mathrm{~min}$; amplification for 30 cycles at $94^{\circ} \mathrm{C}$ for $0.5 \mathrm{~min}$, annealing at $58^{\circ} \mathrm{C}$ for $0.5 \mathrm{~min}$ and extension at $72^{\circ} \mathrm{C}$ for $1 \mathrm{~min}$; followed by a terminal elongation step at $72^{\circ} \mathrm{C}$ for $10 \mathrm{~min}$. The reaction was performed on a CFX96 thermal cycler (Bio-Rad Laboratories, Inc., Hercules, CA, USA). U6 was amplified as an internal control and the $\mathrm{Ct}$ value of each PCR product was calculated, and the fold change analyzed. The $\mathrm{m}$-miR-376b and m-U6 primers were supplied by Ribo Biotechnology (Guangzhou, China) but the sequences were not supplied due to the rules of the company. The results was analyzed using the software that supplied in the CFX-96 (Bio-Rad Laboratories, Inc.)

Luciferase assays. The miR-376b binding site was synthesized and cloned into an pMIR-REPORT vector (Ambion; Thermo Fisher Scientific, Inc.) to generate pMiRluc-376b 3'-UTRs of Hoxd10 containing miR-376b binding sites. These were then amplified and cloned into the same vector to generate pMiRluc-Hoxd10. The reporter was co-transfected with a cytomegalovirus $\beta$-galactosidase vector using FuGENE HD (Promega Corporation, Madison, WI, USA). The luciferase activity was measured $4 \mathrm{~h}$ later using the luciferase reporter assay (\#E1500; Promega Corporation). Values were normalized against $\beta$-galactosidase activity.

Western blot analysis. 4T1 cells were transfected with m-miR-376b-mimic and miR-NC, and $48 \mathrm{~h}$ later the total protein was collected. Cells were lysed on ice for $30 \mathrm{~min}$ with radioimmunoprecipitation assay lysis buffer (Beyotime Institute of Biotechnology, Shanghai, China). The proteins $(20 \mu \mathrm{g})$ were separated by $10 \%$ SDS-PAGE and electronically transferred onto a polyvinylidene difluoride membrane (EMD Millipore, Billerica, MA, USA). Following blocking in $5 \%$ milk in TBS/T buffer for $1 \mathrm{~h}$ at $37^{\circ} \mathrm{C}$, the membranes were incubated with the recommended dilution of primary antibodies against Hoxd10 (\#ab172865; rabbit polyclonal; 1:800 for $1 \mathrm{~h}$ at $37^{\circ} \mathrm{C}$; Abcam, Cambridge, MA, USA), and GAPDH (sc-25778; 1:5,000; Santa Cruz Biotechnology, Inc., Santa Cruz, CA, USA) for $1 \mathrm{~h}$ at $37^{\circ} \mathrm{C}$. This was followed by incubation with goat anti-rabbit (\#ab6721; 1:2,000) and anti-mouse (\#ab6789; 1:2,000) horseradish peroxidase-conjugated secondary antibodies (Abcam) at $1 \mathrm{~h}$ for $37^{\circ} \mathrm{C}$. Peroxidase-labeled bands were visualized using an enhanced chemiluminescence kit (\#32106; Pierce Protein Biology; Thermo Fisher Scientific, Inc., Rockford, IL, USA). The ratio of Hoxd10/GAPDH was then calculated using densitometry, and values were normalized by dividing with the ratio of the blank sample. Protein expression was evaluated using a bicinchoninic acid assay kit (Beyotime, Beijing, China).

Invasion assay. The invasion of 4T1 cells was evaluated using Transwell-24 units (pore size, $8 \mu \mathrm{m}$; EMD Millipore), as described previously with some modification (11). Briefly, the filter of the Transwell plate was covered with $50 \mu 1$ matrigel (BD Biosciences, Franklin Lakes, NJ, USA). Following matrigel polymerization, $500 \mu 1$ DMEM containing 10\% FBS was placed in the lower chamber and $100 \mu 14 \mathrm{~T} 1$ suspension $\left(2 \times 10^{4}\right.$ cells/well in DMEM without $10 \%$ FBS) was placed in the upper chamber. After $48 \mathrm{~h}$ incubation, cells on the back surface were fixed in $100 \%$ methanol, stained with $0.05 \%$ crystal violet, counted in at least six randomly chosen fields and photographed. 
Migration assay. The migration of 4T1 cells was evaluated using Transwell-24 units, as described previously and with some modification. Briefly, $500 \mu$ l DMEM containing $10 \%$ FBS was placed in the lower chamber and a $100 \mu \mathrm{l}$ 4T1 suspension $\left(2 \times 10^{4}\right.$ cells/well; in DMEM without $10 \%$ FBS $)$ was placed in the upper chamber. After a $48 \mathrm{~h}$ incubation, cells on the back surface were fixed in $100 \%$ methanol, stained with $0.05 \%$ crystal violet, counted in at least six randomly chosen fields and photographed (BX600; Olympus Corporation, Tokyo, Japan).

Animal study. Female BALB/c mice (age, 5-6 weeks) were purchased from the Animal Center of Sichuan University (Chengdu, China), with six mice per group. All animal research was approved by the Sichuan Provincial People's Hospital Committee on Animal Research. The mice were housed at $26^{\circ} \mathrm{C}$ under a 12 -h light/dark cycle, with ad libitum access to food and water. The $4 \mathrm{~T} 1$ cell stable transfection with miR-NC and miR-376b-mimic $\left(2 \times 10^{6}\right.$ cells $)$ were injected into the mouse tail vein to establish the 4T1 lung metastasis model. The survival percentage of the mice were analyzed. At 20 days after tumor cell injection, the mice were anesthetized using diethyl ether $(100 \mathrm{mg} / \mathrm{kg}$; Sigma-Aldrich; Merck KGaA, Darmstadt, Germany) then sacrificed by cervical dislocation. The lungs were subsequetly dissected for further study.

$H \& E$ staining. Paraffin-embedded lung sections (3-5 $\mu \mathrm{m})$ were mounted on 3-aminopropyl triethoxysilane-coated glass slides. Sections were deparaffinized in xylene, treated with a graded series of alcohol [100, 95 and $80 \%$ ethanol/double-distilled $\mathrm{H}_{2} \mathrm{O}(\mathrm{v} / \mathrm{v})$ ], and rehydrated in PBS (pH 7.4). Following the PBS washes, the slides were stained with hematoxylin for $3 \mathrm{~min}$ at room temperature followed by washing with $\mathrm{H}_{2} \mathrm{O}_{2}$ for 10-15 min. Next, the slides were stained with eosin for 2-3 min at room temperature followed by washing with $\mathrm{H}_{2} \mathrm{O}_{2}$ for 3-5 min. The slides were then coated with neutral balsam after drying with a graded series of alcohol. All specimens were evaluated using a BX600 microscope (Olympus Corporation) and Spot Fiex camera.

Statistical analysis. Statistical comparisons of all the results were analyzed using a one-way analysis of variance. Statistical analyses were performed using SPSS version 19.0 (IBM SPSS, Armonk, NY, USA). The values are expressed as the mean \pm standard error of the mean. $\mathrm{P}<0.05$ was used to indicate a statistically significant difference.

\section{Results}

Silencing of miR-376b inhibits $4 T 1$ cell migration and invasion in vitro. In order to further determine the role of miR-376b in breast cancer, NBT from BALB/c mice, and mouse breast cancer JC and 4T1 cells were collected for detection of miR-376b expression by qPCR. As shown in Fig. 1A, miR-376b expression in 4T1 cells, which get a high capacity of migration and invasion, was significantly increased compared with NBT $(\mathrm{P}<0.01)$. However, there was no evident difference between NBT and JC on miR-376b expression. The miR-376b-mimic was employed to inhibit the activity of miR-376b. The results indicated that miR-376b expression in 4T1 cells was evidently decreased after being transfected with the miR-376b-mimic (Fig. 1B). Moreover, the migration and invasion capacity of $4 \mathrm{~T} 1$ cells that were transfected with miR-NC or the miR-376b-mimic were determined by transwell migration assay and transwell-based matrigel invasion assay. The results shown in Fig. 1C and D indicated that silencing of miR-376b significantly inhibited 4T1 cell migration and invasion. Overall, the results of the present study demonstrated that miR-376b was important in regulating breast cancer migration and invasion in vitro.

Hoxd10 is a direct target of miR-376b. In order to determine the targets of miR-376b, a large number of potential target proteins were screened in a database library to identify promising miRNA binding seed sequences within the 3'-UTR. Using bioinformatics analysis tools (Targetscan.org, RNA.org and microRNA Seq), Hoxd10 was identified as a candidate target for miR-376b (Fig. 2A). In order to verify this result, Hoxd10-3'UTR containing an miR-376b binding site was cloned downstream of the luciferase open reading frame. Meanwhile, a Hoxd10-3'UTR mutant, which contained a mutated miR-376b binding site, was also introduced into the luciferase construct. The plasmid expressing miR-376b was transfected in the 293 cells, and puromycin was used to select the stable expression cells. qPCR analysis confirmed that miR-376b was upregulated in the cells (Fig. 2B). Next, the luciferase-Hoxd10-3'UTR and luciferase-Hoxd10-3'UTR mutant constructs were transfected into 293 cells for stable miR-376b expression. After 4-6 h, luciferase expression in Hoxd10-3'UTR constructs was significantly reduced compared with the control (Fig. 2C). Moreover, a consistent reduction of luciferase expression was observed in cells transfected with miRNA binding site mutant plasmids (Fig. 2C). Further western blotting indicated that Hoxd10 expression in 4T1 cells was significantly increased after being transfected with the miR-376b-mimic (Fig. 2D). The results described above demonstrated that Hoxd10 is a direct target of miR-376b.

Silencing of miR-376b inhibits $4 T 1$ cell metastasis in mice. In order to establish a breast cancer metastasis model, $2 \times 10^{6} 4 \mathrm{~T} 1$ cells that were stably transfected with miR-NC or the miR-376b-mimic, were intravenously injected into $\mathrm{Balb} / \mathrm{c}$ mice separately. The results of the present study indicated that silencing of miR-376b after transfection with the miR-376b-mimic significantly increased the survival percentage, compared with the miR-NC transfected group (Fig. 3A). In total, 7 out of 10 mice died in the miR-NC transfected group, whereas only 1 out of 10 mice died in the miR-376b-mimic transfected group at 20 days post cancer cell injection (Fig. 3A). Bright-field microscopy and H\&E staining demonstrated that less lung metastasis was found in the miR-376b-mimic transfected group compared to the miR-NC transfected group (Fig. 3B and C). In total, a 69\% decrease in the number of macroscopically visible pulmonary metastasis was achieved through silencing of miR-376b ( $\mathrm{P}=0.001)$ (Fig. 3D). Moreover, a smaller tumor area (tumor area/total lung area) was observed in the miR-376b-mimic compared to the miR-NC transfected group (Fig. 3E). Overall, 4T1 cell metastasis in mice was significantly inhibited after silencing miR-376b. 
A

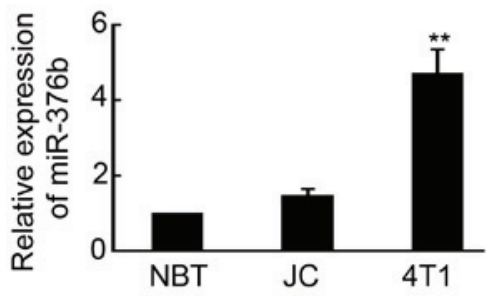

C

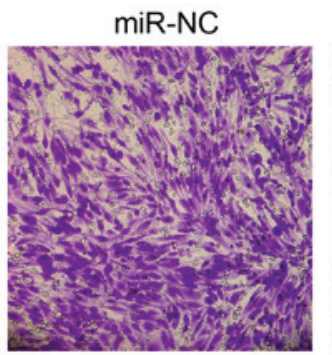

D

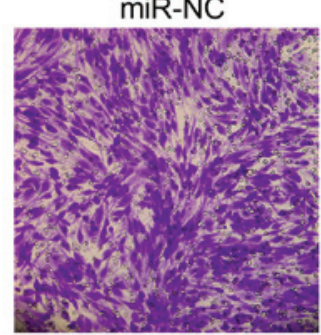

miR-376b-mimic

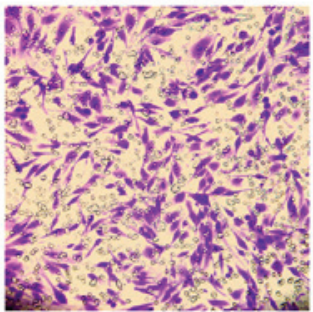

miR-376b-mimic

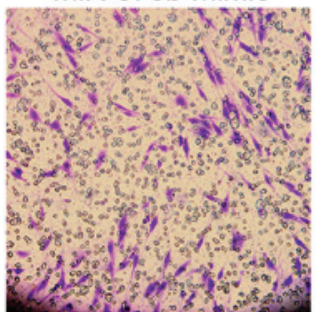

B
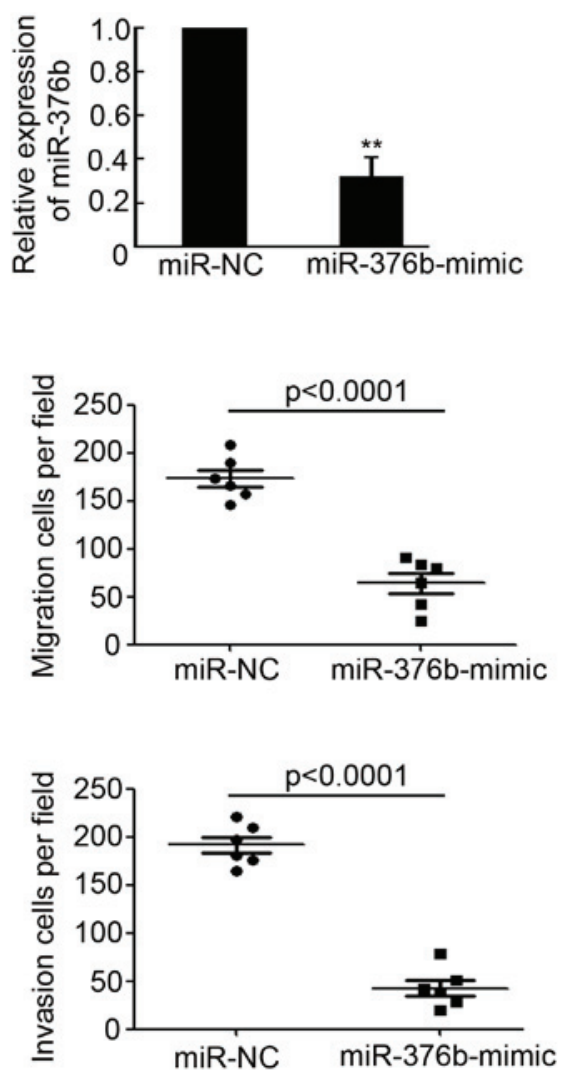

Figure 1. Silencing of miR-376b inhibits $4 \mathrm{~T} 1$ cell migration and invasion. (A) miR-376b expression was detected in normal breast tissue from mice and JC and $4 \mathrm{~T} 1$ breast cancer cells using quantitative polymerase chain reaction (qPCR; $n=3 ;{ }^{* * *} \mathrm{P}<0.01$ ). (B) miR-376b expression in $4 \mathrm{~T} 1$ cells after transfection with miR-NC and miR-376b-mimic for $48 \mathrm{~h}$ was detected by qPCR $\left(\mathrm{n}=3 ;{ }^{* * *} \mathrm{P}<0.01\right)$. (C) Transwell migration assay of $4 \mathrm{~T} 1$ cells that were transfected with miR-NC or miR-376b-mimic. The migrated cells per field were analyzed $(n=6)$. (D) Transwell-based matrigel invasion assay of 4T1 cells that were transfected with miR-NC or miR-376b-mimic. The invaded cells per field were analyzed $(n=6)$. miR, microRNA; NC, normal control.

A

\begin{tabular}{|c|}
\hline 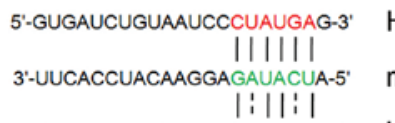 \\
\hline
\end{tabular}

5'-GUgaucuguaAucccuaugag-3' Hoxd10 3'UTR mutant

C

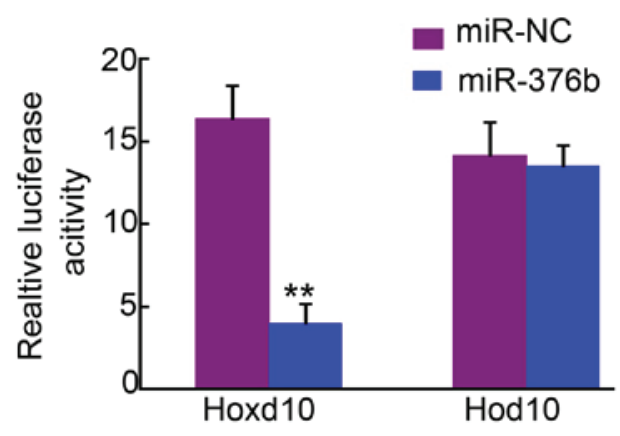

B

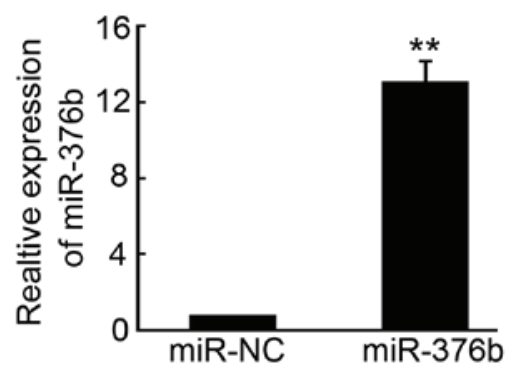

D

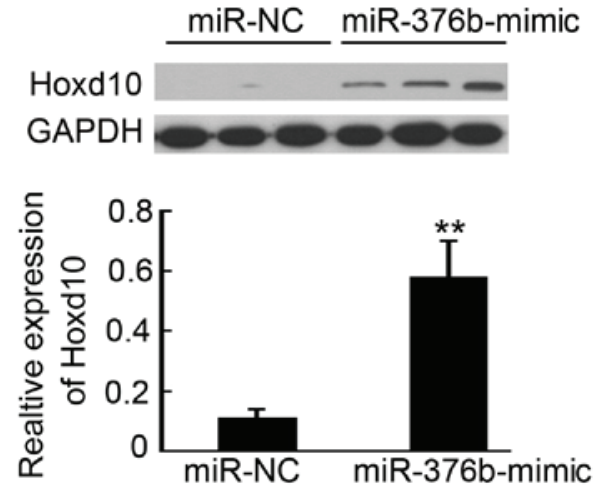

Figure 2. Hoxd10 is a direct target of miR-376b. (A) Hoxd10 3'-UTR contains one predicted miR-376b binding site. The figure shows predicted duplex formations between Hoxd10 3'UTR and miR-376b. The sites of target mutagenesis are indicated in blue. (B) Quantitative polymerase chain reaction analysis of miR-376b in 293 cells after enforced expression of miR-376b in 293 cells. (C) Relative repression of luciferase expression was standardized to a transfection control ( $\mathrm{n}=4 ;{ }^{* *} \mathrm{P}<0.01$ vs. miR-NC control). (D) Hoxd10 expression in 4T1 cells that transfected with miR-NC or miR-376b-mimic was detected by western blot analysis. The density of Hoxd10/GAPDH was analyzed ( $\left.\mathrm{n}=3 ;{ }^{* * *} \mathrm{P}<0.01\right)$. UTR, untranslated region; miR, microRNA; NC, normal control. 


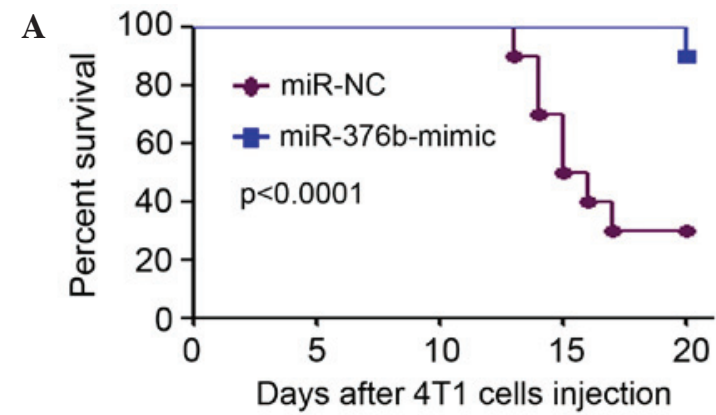

B

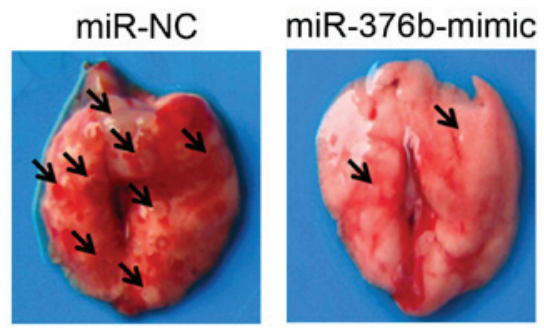

C

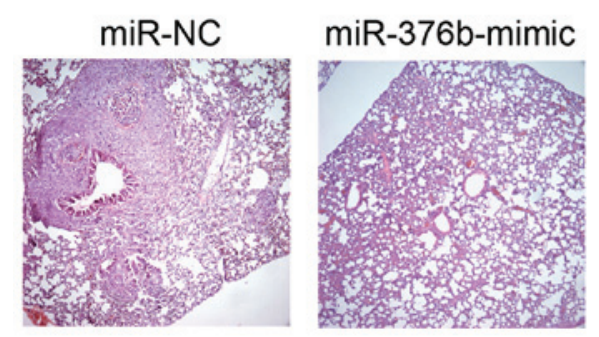

D

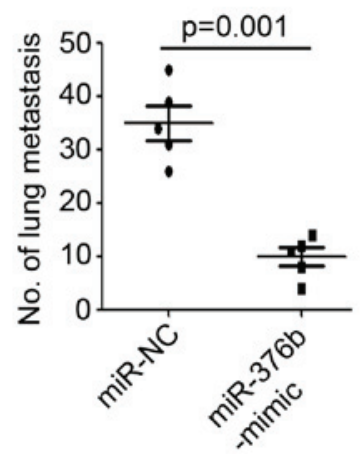

$\mathbf{E}$

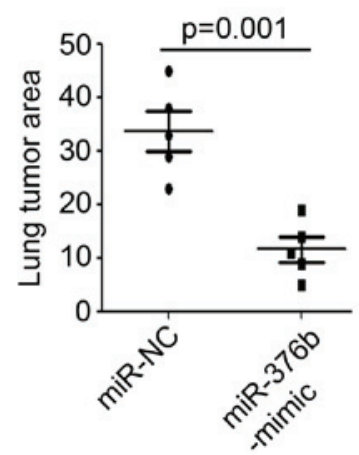

Figure 3. Silencing of miR-376b inhibits 4T1 cell metastasis in mice. (A) Survival percent of the mice intravenous injected with miR-NC or miR-376b-mimic transfected 4T1 cells ( $\mathrm{n}=10 ; \mathrm{P}<0.0001)$. (B) Bright imaging of the lungs from 4T1 bearing mice. (C) Hematoxylin and eosin staining was used to test the metastasis of the lungs (magnification, x200). Arrows indicate lung metastasis. (D and E) Number of visible lung metastases and tumor area in lungs in 4T1-bearing mice (miR-NC or miR-376b-mimic transfected 4T1 cells; $\mathrm{n}=5, \mathrm{P}=0.001$ ). miR, microRNA; NC, normal control.

A

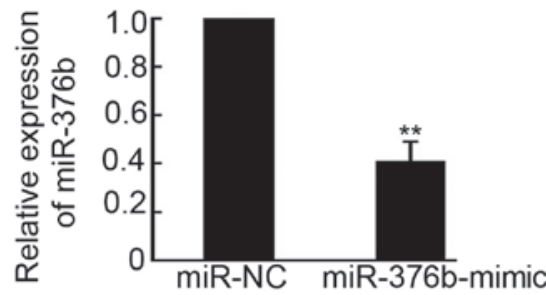

B

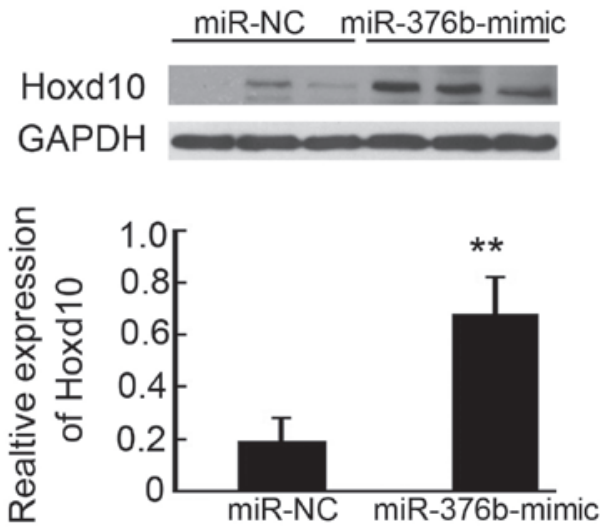

Figure 4. Silencing of miR-376b and inducing of Hoxd10 in lung of mice. (A) miR-376b expression in the lungs of 4T1-bearing mice (miR-NC or miR-376b-mimic transfected 4T1 cells) was detected using quantitative polymerase chain reaction analysis ( $\left.\mathrm{n}=5 ;{ }^{* *} \mathrm{P}<0.01\right)$. (B) Hoxd10 expression in the lungs of 4T1-bearing mice (miR-NC or miR-376b-mimic transfected $4 \mathrm{~T} 1$ cells) was detected by western blot analysis. The density of Hoxd10/GAPDH was analyzed ( $\mathrm{n}=3 ;{ }^{* *} \mathrm{P}<0.01$ ). miR, microRNA; NC, normal control.

Silencing of miR-376b and induction of Hoxd10 in the lungs of mice. Relative to the effects of the miR-376b-mimic on decreasing 4T1 metastasis in mice, miR-376b and Hoxd10 expression in the lungs after 4T1 cancer cell injection was determined by qPCR and western blotting. As shown in Fig. 4A, the mice injected with the miR-376b-mimic transfected 4T1 cells exhibited a $61 \%$ reduction of $\operatorname{miR}-376 \mathrm{~b}$ expression in the lungs, compared with miR-NC transfected group. Meanwhile, Hoxd10 expression was upregulated 3.6-fold after silencing miR-376b (Fig. 4B). These results are consistent with the observation that silencing of miR-376b inhibited $4 \mathrm{~T} 1$ cell metastasis in mice.

\section{Discussion}

The present study initially examined the expression of miR-376b in NBT, JC and 4T1 breast cancer cells by qPCR. The results indicated that miR-376b was highly expressed in 4T1 cells, which gets the high capacity of migration and invasion, compared with normal mouse breast tissue $(8,12)$. Further migration and invasion assays demonstrated that silencing of miR-376b significantly inhibited 4T1 cell migration and invasion in vitro. Lung metastasis was also evidently decreased after silencing of miR-376b in 4T1 cells. Luciferase assay and western blotting identified that Hoxd10 is the direct target of 
miR-376b during the regulation of breast cancer metastasis. To the best of our knowledge, the present study was the first to demonstrate the promoting breast cancer metastasis role of miR-376b.

Hoxd10 belongs to the type I class homeobox (Hox) family, and is important for suppressing angiogenesis and maintaining a quiescent, differentiated phenotype in endothelial cells (13). In particular, Hoxd10 suppresses expression of genes that directly affect remodeling of the extracellular matrix and cell migration during angiogenesis, including $\alpha 3$ integrin, matrix metalloproteinase 14 and urokinase-type plasminogen activator receptor (14). A previous study has indicated that Hoxd10 inhibits the development and progression of head and neck squamous cell carcinoma (15). Meanwhile, various studies have demonstrated that Hoxd10 was involved in the metastasis of breast, bladder, gastric and hepatocellular cancer (16-19). In the present study, a luciferase assay was performed that indicated that Hoxd10 is the direct target of miR-376b. Silencing of miR-376b in 4T1 cells significantly induced Hoxd10 expression both in vitro and in vivo. Meanwhile, inducing expression of Hoxd10 by miR-376b-mimic significantly inhibited 4T1 cell migration and invasion in vitro and lung metastasis in vivo.

The 4T1 cell line was originally derived from a spontaneous mouse mammary carcinoma from the BALB/c strain (20). A previous study has reported that $4 \mathrm{~T} 1$ cells get a high capacity of migration and invasion $(8,12)$. The intravenous injection of 4T1 cells into the BALB/c mice could form metastasis in the lungs. Therefore, a 4T1 breast mouse model is appropriate to mimic human breast cancer metastasis in vivo, and for investigating the effect of miR-376b on metastasis. In the present study, the miR-NC or miR-376b-mimic transfected 4T1 cells was injected into the mouse tail vein. At 20 days post $4 \mathrm{~T} 1$ cell injection, the survival percentage, lung metastasis formation number and lung metastasis area were analyzed. The results indicated the metastasis capacity of miR-376b in vivo. It is consistent with the results in the migration and invasion assays. Moreover, lower expression of miR-376b and higher expression of Hoxd10 were also observed in the lungs from the miR-376b-mimic transfected group.

In summary, the present study initially demonstrated the role of miR-376b in regulating breast cancer metastasis in vitro and in vivo. Moreover, the luciferase assay indicated that Hoxd10 is the direct target of miR-376b during the regulation of breast cancer metastasis. Thus, these results may provide a novel therapy target and prognosis biomarker for breast cancer.

\section{References}

1. Siegel R, Naishadham D and Jemal A: Cancer statistics, 2013. CA Cancer J Clin 63: 11-30, 2013.
2. Fidler IJ: The pathogenesis of cancer metastasis: The 'seed and soil' hypothesis revisited. Nat Rev Cancer 3: 453-458, 2003.

3. Lee YT: Breast carcinoma: Pattern of metastasis at autopsy. J Surg Oncol 23: 175-180, 1983.

4. Steeg PS: Tumor metastasis: Mechanistic insights and clinical challenges. Nat Med 12: 895-904, 2006.

5. Baek D, Villén J, Shin C, Camargo FD, Gygi SP and Bartel DP: The impact of microRNAs on protein output. Nature 455: 64-71, 2008.

6. Ambros V: The functions of animal microRNAs. Nature 431: 350-355, 2004.

7. van Rooij E, Marshall WS and Olson EN: Toward microRNA-based therapeutics for heart disease: The sense in antisense. Circ Res 103: 919-928, 2008.

8. Ma L, Reinhardt F, Pan E, Soutschek J, Bhat B, Marcusson EG, Teruya-Feldstein J, Bell GW and Weinberg RA: Therapeutic silencing of miR-10b inhibits metastasis in a mouse mammary tumor model. Nat Biotechnol 28: 341-347, 2010.

9. Li LJ, Huang Q, Zhang N, Wang GB and Liu YH: miR-376b-5p regulates angiogenesis in cerebral ischemia. Mol Med Rep 10: 527-535, 2014.

10. Pan Z, Guo Y, Qi H, Fan K, Wang S, Zhao H, Fan Y, Xie J, Guo F, Hou Y, et al: M3 subtype of muscarinic acetylcholine receptor promotes cardioprotection via the suppression of miR-376b-5p. PLoS One 7: e32571, 2012.

11. Dai L, Cui X, Zhang X, Cheng L, Liu Y, Yang Y, Fan P, Wang Q, Lin Y, Zhang J, et al: SARI inhibits angiogenesis and tumour growth of human colon cancer through directly targeting ceruloplasmin. Nat Commun 7: 11996, 2016

12. Cronin PA, Wang JH and Redmond HP: Hypoxia increases the metastatic ability of breast cancer cells via upregulation of CXCR4. BMC Cancer 10: 225, 2010.

13. Myers C, Charboneau A, Cheung I, Hanks D and Boudreau N: Sustained expression of homeobox D10 inhibits angiogenesis. Am J Pathol 161: 2099-2109, 2002.

14. Sekar P, Bharti JN, Nigam JS, Sharma A and Soni PB: Evaluation of p53, HoxD10 and E-Cadherin status in breast cancer and correlation with histological grade and other prognostic factors. J Oncol 2014: 702527, 2014.

15. Hakami F, Darda L, Stafford P, Woll P, Lambert DW and Hunter KD: The roles of HOXD10 in the development and progression of head and neck squamous cell carcinoma (HNSCC). Br J Cancer 111: 807-816, 2014.

16. Li Q, Ding C, Chen C, Zhang Z, Xiao H, Xie F, Lei L, Chen Y, Mao B, Jiang M, et al: miR-224 promotion of cell migration and invasion by targeting Homeobox D 10 gene in human hepatocellular carcinoma. J Gastroenterol Hepatol 29: 835-842, 2014.

17. Liu Z, Zhu J, Cao H, Ren H and Fang X: miR-10b promotes cell invasion through RhoC-AKT signaling pathway by targeting HOXD10 in gastric cancer. Int J Oncol 40: 1553-1560, 2012

18. Vardhini NV, Rao PJ, Murthy PB and Sudhakar G: HOXD10 expression in human breast cancer. Tumour Biol 35: 10855-10860, 2014.

19. Xiao H, Li H, Yu G, Xiao W, Hu J, Tang K, Zeng J, He W, Zeng G, Ye Z and Xu H: MicroRNA-10b promotes migration and invasion through KLF4 and HOXD10 in human bladder cancer. Oncol Rep 31: 1832-1838, 2014

20. Pulaski BA, Terman DS, Khan S, Muller E and Ostrand-Rosenberg S: Cooperativity of staphylococcal aureus enterotoxin B superantigen, major histocompatibility complex class II, and CD80 for immunotherapy of advanced spontaneous metastases in a clinically relevant postoperative mouse breast cancer model. Cancer Res 60: 2710-2715, 2000. 